\title{
Actuator selection for variable camber foils
}

\author{
John D. Madden \\ Dept. of Electrical and Computer Engineering, The University of British Columbia, \\ 2356 Main Mall, Vancouver, BC Canada V6T 1Z4, jmadden@ ece.ubc.ca.
}

\begin{abstract}
A number of polymer based actuator technologies have emerged over the past decade. How do these compare with traditional actuators and are there applications for which they are appropriate? Some of the answers to these questions are provided by outlining the rationale for employing an electroactive polymer to control hydrodynamic surfaces. The surfaces are sections of propeller blades whose trailing edges are deflected in order to change camber. The objective is to insert the actuators into the blades. High work per unit volume is required of the actuators. The ideal actuator technologies also feature relatively large strains in order to deflect the trailing edges with minimal mechanical amplification. It is argued that the high work densities, flexibility in shaping and the ability to hold a force without expending energy (catch state) provide electroactive polymers with advantages over electromagnetic actuators, which also lack the torque to directly drive the blade deflection. Candidate actuators are compared, including electroactive polymers, shape memory alloys, magnetostrictives and traditional piezoceramics. Selections are made on the bases of work density, strain, existence of a catch state, drive voltage and cost. It is suggested that conducting polymer actuators are best suited for the variable camber application. It is also argued that in general electroactive polymers are wellsuited for applications in which actuator volume or mass are very limited, catch states are desired, cycle life is moderate to low, or noise cannot be tolerated. Some electroactive polymers also feature low voltage operation, and may be biocompatible.
\end{abstract}

Keywords: Actuator, Autonomous Underwater Vehicle, Artificial Muscle, Conducting Polymer, Polypyrrole, Dielectric Elastomer, Ferroelectric Polymer, Motor, Shape Memory Alloy.

\section{INTRODUCTION}

In this paper the feasibility of using conducting polymer actuators to vary the camber of propeller blades in autonomous underwater vehicles is investigated. Although the application is very specific, the experiments and analysis performed here provide guidance in the general design issues and concerns encountered in applying electroactive polymers.

New actuator materials [1] such as dielectric elastomers, ferroelectric polymers, conducting polymers and carbon nanotubes can offer power to mass ratios that are within a factor of 4 of the combustion engine and high revving electric motors $[2,3,4]$. Although the specific power outputs of these materials are lower, these may be compensated for by their 'muscle-like' natures, making them more suitable for biomimetic propulsion [5,6,7] among other applications. For example, discontinuous and aperiodic motions such as the grasping of parts by a robot arm, the opening of a valve or the adjustment of a hydrodynamic control surface are not easily performed using rapidly spinning actuators with relatively narrow ranges of optimal rotation rates, such as the combustion engine or high revving electric motors. In such cases direct drive electric motors are often employed. However the relatively low force, torque and work to mass ratios of these direct drive actuators compared to muscle and many emerging actuator technologies adds a weight and volume penalty to their use $[2,3,4]$. A further disadvantage of the electromagnetic actuators is that they expend energy to hold a force, even when no mechanical work is being performed. Holding a fixed position under load is thus highly inefficient, unless a catch, brake or lock can be implemented. Applications of a discontinuous nature occurring in situations where the space and or mass are at a premium could benefit from actuators with high force and work densities that also feature catch states. One such application which will be examined further is the positioning of hydrodynamic surfaces in order to change the camber of propeller blades in autonomous underwater vehicles. 
Deflection of the leading and trailing edges of a hydrofoil, such as a propeller blade, can lead to large changes in loading resulting from only small input forces [8]. Potential advantages of varying blade geometry are similar to those afforded by variable pitch blades and include better efficiency at offdesign speeds, an ability to adjust load to match engine or motor characteristics, reduced cavitation, improved low speed maneuverability, and thrust reversal without reversing shaft rotation direction [8]. By placing an electrically controllable actuator directly within the propeller blade torque can be optimized over a wide range of speeds without the use of gearboxes, and no special transmission is required (unlike with variable pitch propellers, whose actuation is
Span $50 \mathrm{~mm}$

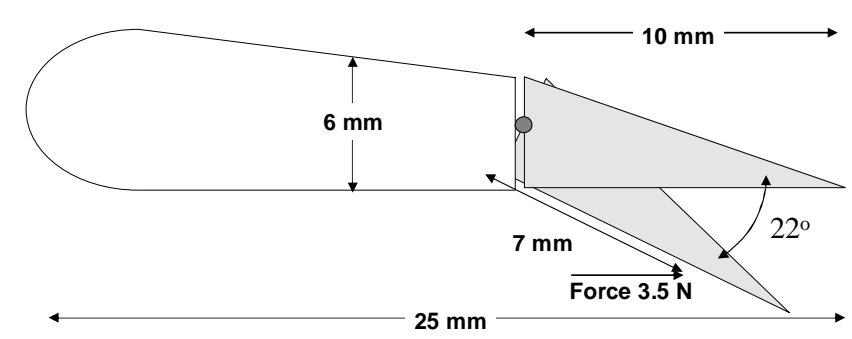

Available Volume $<4.5 \times 10^{-6} \mathrm{~m}^{3}$

Work $19 \mathrm{~mJ}$

Work Density $>8 \mathrm{~kJ} / \mathrm{m}^{3}$

Time Response $1-10 \mathrm{~s}$

Power 2-20 mW

Power Density $<50 \mathrm{~kW} / \mathrm{m}^{3}$ or $<35 \mathrm{~W} / \mathrm{kg}$

Figure 1: Blade Geometry. A cross-sectional view of a single propeller blade is shown, indicating the geometrical changes, forces and work needed to effect variable camber. The leading section (portion to the left of the pivot point) is assumed to have free volume available within.

transmitted down the shaft). The mechanical complexity and cost of the pitch control mechanism is the major disadvantage of such propellers, since it requires gearing in the hub for each blade as well as control rods embedded in the propeller shaft and actuators inside the ship, needing much more complex sealing than a simple shaft [8]. Such systems are relatively expensive in comparison to fixed pitch propellers, and require more maintenance to ensure reliability [8]. For these reasons pitch control is primarily limited to large vessels. An objective of this work is to extend the advantages afforded by variable pitch to small, low cost autonomous underwater vehicles. New performance advantages may also result. For example, blades can also controlled individually, potentially providing increased maneuverability and noise reduction.

Efforts are underway to experimentally quantify the benefits of variable camber propulsion [8]. The aim of this paper is to review the process of actuator selection. In particular the following questions are addressed: Are electraoctive polymers, and polypyrrole in particular, appropriate actuator technologies for such applications and what are the advantages, disadvantages and challenges compared to alternatives?

\section{SPECIFICATIONS}

The target vehicle for variable camber is EMATT (Expendable, Mobile Anti-submarine warfare Training Target from Sippican Inc, in Marion, Massachusetts [9]). The vehicle is designed to act as a target for antisubmarine warfare exercises. Based on the estimated EMATT properties minimum performance specifications that must be reached by the actuator/propulsor combination are determined, as described in detail in reference 8 . With three blades per propeller and operating at the maximum speed of $4 \mathrm{~m} / \mathrm{s}$, the upper load limit is $7 \mathrm{~N}$ per blade, acting over a span of $50 \mathrm{~mm}$. Less than $3.5 \mathrm{~N}$ per blade is born by the trailing edge. This is the maximum force that must be countered by an actuator controlling blade camber [8]. A trailing edge deflection of up to \pm 22 degrees occurring on the last 25-40 percent of the chord is selected as being a common high-lift flap geometry [8]. EMATT is a single use vehicle. The device must operate over a time period of 24 hours without failure (the maximum mission duration). Assuming that camber variations are needed at most once every minute on average, the upper bound on number of cycles sustained by the moveable flap is 1500 . The EMATT performs shallow dives, attaining relative pressures of up to $\sim 2 \mathrm{MPa}$. The vehicle must also be able to withstand a corrosive aqueous environment. The cost of the variable camber mechanism should be on the order of $\$ 100$ or less. Fifteen DD size primary Lithium-Sulfur dioxide batteries with nominal voltages of $3 \mathrm{~V}$ provide a total energy total of $3 \mathrm{MJ}$ for the entire vessel. 


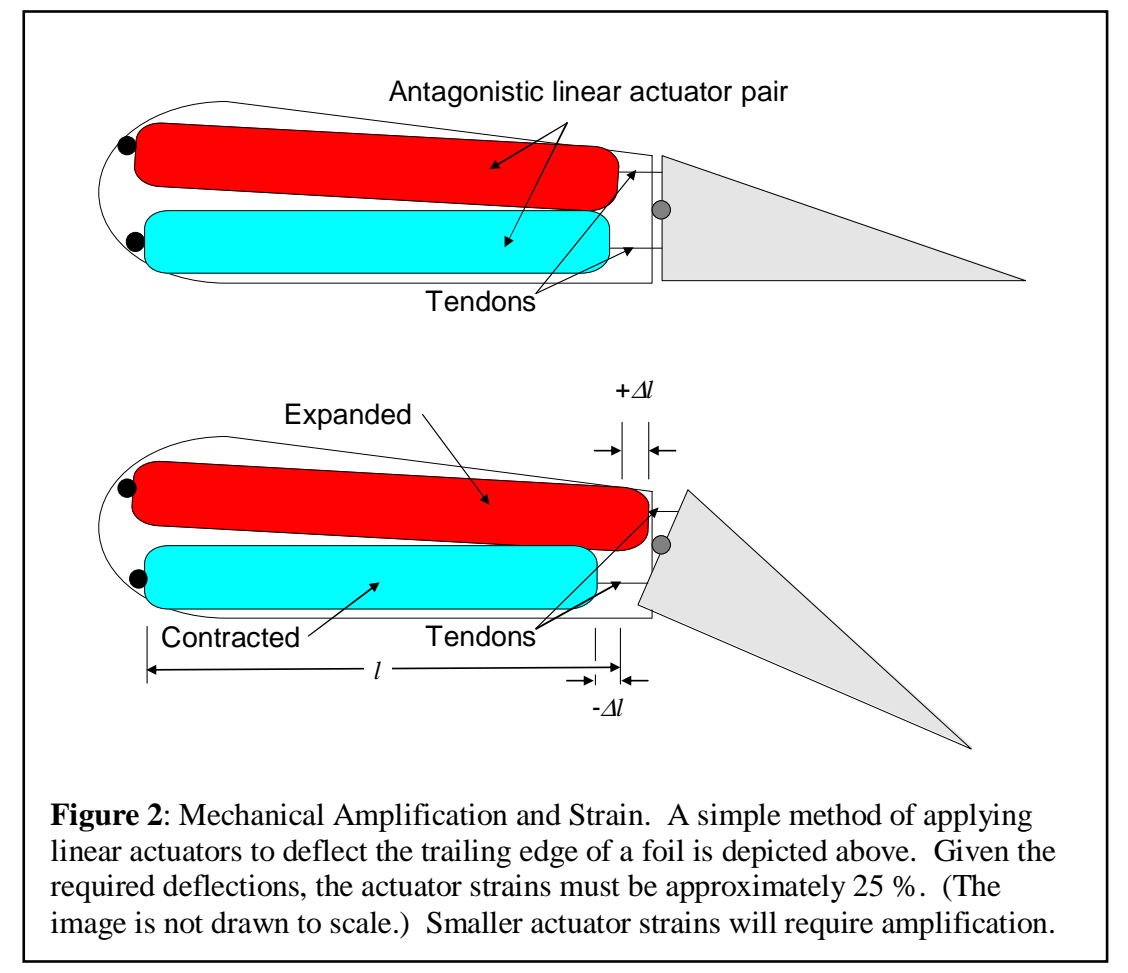

Actuator Requirements: The next step is to calculate the forces, displacements, work densities, rates and cycle life required of the actuators. Each EMATT propeller blade has an approximate span (length) of $50 \mathrm{~mm}$, a chord (width) of $25 \mathrm{~mm}$, and an average thickness of 6 $\mathrm{mm}$, as depicted in Figure 1. In the proposed design the last $10 \mathrm{~mm}$ of the chord at the trailing edge will be pivoted so as to enable variable camber. Figure 2 shows the approximate geometry. The angle of the trailing edge is to be adjusted by $\pm 22^{\circ}$ under a load of up to $3.5 \mathrm{~N}$. The peak torque that must be generated to maintain the required forces is $T=25 \mathrm{mN} \cdot \mathrm{m}$ about the pivot. The upper bound on work is $19 \mathrm{~mJ}$ per cycle, and the available volume within the blade is $4.5 \times 10^{-6} \mathrm{~m}^{3}$. Leaving space for connections, sensors, packaging and the structure itself, the work density of the actuator (assuming one actuator stroke generates a full trailing edge deflection) is $>8 \mathrm{~kJ} \cdot \mathrm{m}^{-3}$. Assuming adjustment of the camber takes place over $1 \mathrm{~s}$ or longer, the output power is at most $19 \mathrm{~mW}$.

\section{ACTUATOR SELECTION \& CHALLENGES}

One can choose to vary the camber via a mechanism that extends down the propeller shaft, or to place the actuator in the blade itself. The second option appears simpler and more cost effective on first examination, and the ultimate object is to determine the feasibility of such an approach. In proceeding to answer this question it is first shown that direct drive electric motors are not well suited for insertion into a blade due to their relatively low torque and their lack of a catch state. The range of actuator options are then compared based on a series of criteria including their work densities, actuator strains, applied potentials, costs and the availability of catch states. Experimental investigations of the feasibility are reported elsewhere [8].

DC Servo Motors: Direct drive electric motors are a well established, commercially available technology. These are powered by DC voltages readily obtained from batteries. There is a strong incentive to employ such established technology where possible. However these do not exhibit sufficient torque given the available volume for direct drive application. For example the Futaba S3101 micro servo [10] has dimensions of $28 \mathrm{~mm} \times 12.7 \mathrm{~mm} \times 30 \mathrm{~mm}$, a mass of 17 grams and with $4.8 \mathrm{~V}$ applied generates a torque of $25 \mathrm{mN} \cdot \mathrm{m}$ (exactly the required torque). Unfortunately the volume is twice as large as the maximum available within each blade, and the cross-section is square rather than being elongated. Not only is the torque too small, but the work per volume, assuming a rotation of $44^{\circ}$, is approximately 2 $\mathrm{kJ} / \mathrm{m}^{3}$, much lower than the minimum $8 \mathrm{~kJ} / \mathrm{m}^{3}$ that is specified. Finally, there is no actuator catch state, and thus energy is continually being expended when the blade is held at a constant position.

Custom motors optimized for torque production have attained torque to mass ratios of $10 \mathrm{~N} \cdot \mathrm{m} / \mathrm{kg}$ [11], about 5 times greater than are observed in conventional motors [10], but these suffer from a number of drawbacks. As with the Futaba motors, a catch mechanism or servo control is needed to maintain position. The parallel disk geometry of these custom motors is even worse than the tetragonal shape of most motors for making effective use of the hydrofoil volume, they are not commercially available, and are challenging to build due to their magnetically unstable configuration [11]. For these reasons alternative actuator technologies are considered. 


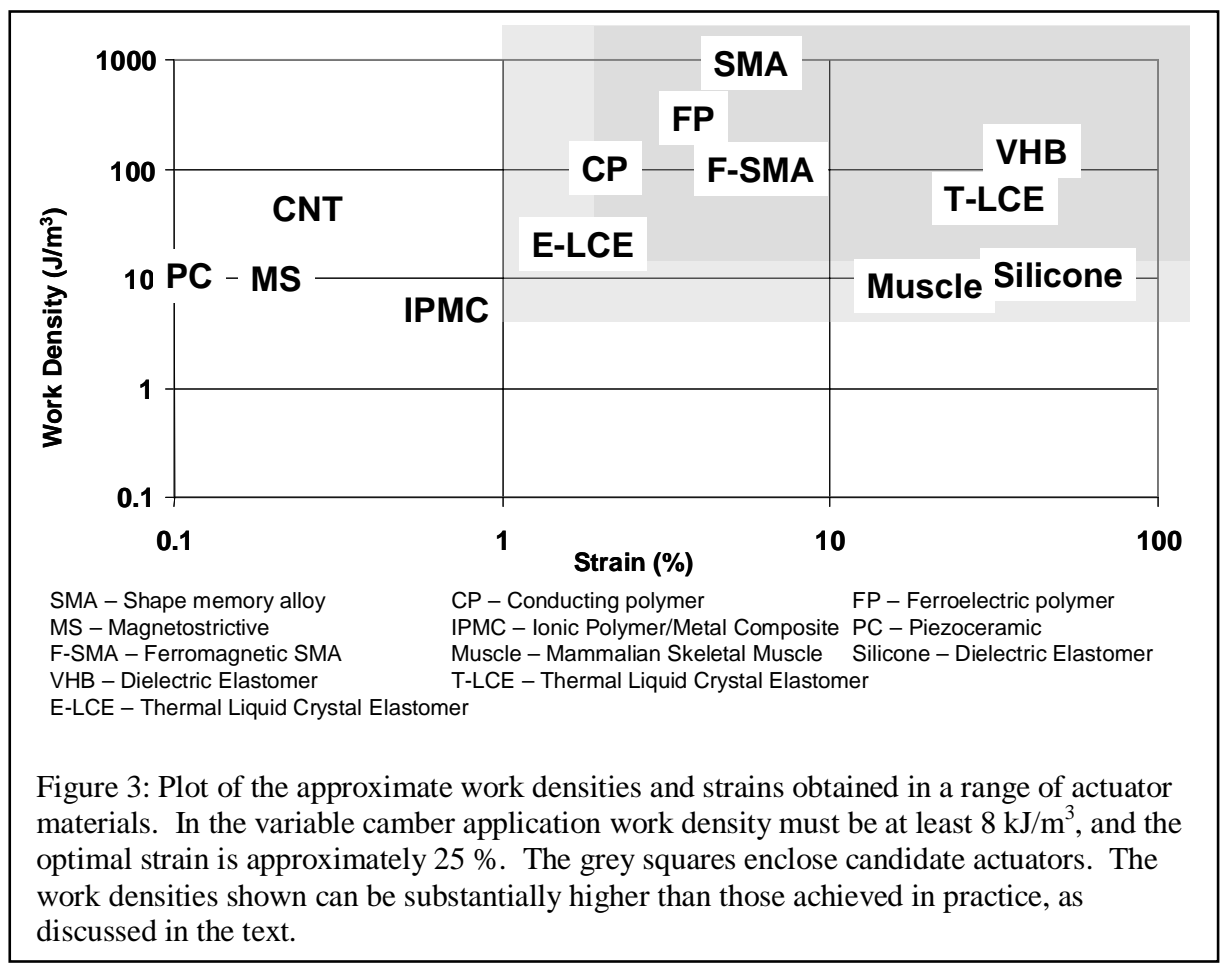

Work Density and Mechanical Amplification: In selecting appropriate actuator technologies a key question immediately arises: will any actuators fit within the confined space of the propeller blade, while still generating sufficient force and work to perform the task? Available space and allowable mass are generally limited, and therefore this question arises in nearly all design cases. A further consideration is the mechanical amplification needed to transform the displacements generated by a given actuator technology into the specified device rotations or translations. For example, in the variable camber foil a $22^{\circ}$ rotation is required. If a pair of linear actuators is employed to drive the trailing edge angle, as depicted in Figure 2, then for the given geometry, the actuator strain must be on the order of $25 \%$. Smaller strains must be amplified, adding to the complexity of the device, with increasing complexity required the larger the amplification factor. The ideal actuator technology will have very high work density, and feature strains that are on the order of $25 \%$. These key metrics are employed as a basis for comparing actuator technologies. A plot of typical work densities and strains for a number of established and emerging actuator materials is shown in Figure 3 . In principle the most suitable actuator technologies feature large strains, and high work density, which, as specified above, must be at least $8 \mathrm{~kJ} / \mathrm{m}^{3}$. There are a number of technologies that are close to meeting the requirements. All of the technologies shown in Figure 3 are now discussed briefly, the positive features identified and the limitations mentioned.

Piezoceramics (PC). Piezoelectric materials feature high power to mass and large bandwidth [4]. A challenge with piezoelectrics is to transform their small strains, which are typically on the order of $0.1 \%$ [4], into large displacements. Small strains have kept this established class of materials from competing with small electric motors, despite offering higher specific power, and for the same reason they are not well suited for the present application. Newer material combinations are enabling strains on the order of $1 \%$ and may prove useful for moderate displacement applications.

Magnetostrictive Materials (MS). Like piezoceramics, giant magnetostrictive materials feature high specific power and bandwidth [4]. Unlike piezoceramics, voltages can be very low since actuation involves varying magnetic fields. Low voltages are attractive given the battery power commonly available onboard autonomous underwater vehicles. However, strains are small $(<0.2 \%)$, and the work density quoted is reduced by more than an order of magnitude when the coils necessary for field generation are accounted for, making it impossible to fit the actuator within the desired space.

Ferromagnetic Shape Memory Alloys (F-SMA). F-SMAs feature strains in the percent range [2], a substantial improvement over those of giant magnetostrictive materials [4]. However, the work density drops to less than $1 \mathrm{~kJ} / \mathrm{m}^{3}$ when the volume of the field generating coils is accounted for. The effective work density is well below the requirements of the application being considered.

Carbon Nanotube Actuators (CNT). Carbon nanotube-based actuators promise to enable unprecedented stresses (gigaPascal level) and work densities [2]. They have the further advantage of operating at low voltages $(\sim 2 \mathrm{~V})$. Currently these materials are expensive however and the strains are small $(<1 \%)$ [2]. This technology promises to evolve substantially, but is not yet ready for the variable camber application. 
Ionic Polymer/Metal Composites (IPMC). IPMCs actuate at low voltages $(<10 \mathrm{~V})$ and generate large deflections. Mechanical amplification is built in, as these materials form a bilayer-like configuration which bends upon application of voltage [2]. An important disadvantage of IPMCs for this particular application is that they generally do not feature a catch state, with energy continually dissipated in order to maintain a fixed position under constant load. Maintaining a fixed position is a further challenge as these materials tend to relax back towards their initial state.

Shape Memory Alloys (SMA). Thermally activated shape memory alloys feature unprecedented work and power densities [2,4], along with moderate to large strains. Unfortunately however the maintenance of fixed position requires continuous heating and thus there is no catch state. Furthermore SMAs are difficult to position control beyond a simple all or nothing response, making continuous activation of control surfaces challenging [2,4].

Thermally actuated Liquid Crystal Elastomers (T-LCE). These emerging materials feature excellent strains and work densities [2]. Like shape memory alloys, T-LCEs undergo a temperature driven phase change. Continuous heating is generally required and, as in SMAs, intermediate control of position is challenging.

Mammalian Skeletal Muscle. The use of muscle as an engineering material [4] is intriguing given its elegant properties. Obstacles to the use of muscle include challenges in control and the maintenance of viability. A circulation system also needs to be maintained.

Eight candidate actuator technologies have been eliminated from consideration for reasons including small strain, lack of a catch state, poor controllability and low effective work density. Four technologies remain, namely electrically actuated liquid crystal elastomers (E-LCE), dielectric elastomers (VHB and Silicone), relaxor ferroelectric polymers (FP) and conducting polymer actuators (CP).

Work Density Revisited. All the technologies feature substantially larger work densities than are required. This margin is somewhat misleading however, as the work is normalized by the volume of actuator material alone. In conducting polymers electrolyte and a counter electrode are required, for example, leading to a reduction in the effective work density, the extent of which is very much a function of the design employed. VHB and silicone dielectric elastomers are generally employed in a pre-stretched state in order to amplify strain and improve properties. The prestretching requires mechanical mechanisms which add substantial mass, often reducing the effective work density by an order of magnitude [12]. Given these considerations, the silicone based dielectric elastomer is impractical .

Strain and Mechanical Amplification. Strains vary between the actuator technologies from $2 \%$ to greater than $30 \%$. Smaller strains generally are observed in stiffer materials, which can withstand higher forces, thereby enabling high work densities despite small strains. However strains of less than $25 \%$ will need mechanical amplification in order to generate the displacements needed in the variable camber propeller. Implementation with VHB-based dielectric elastomer thus appears to be the simplest approach, and, given the simplicity combined with the ready availability of the actuating material, this is seemingly the most cost-effective approach.

Voltage and Cost. A final consideration is the voltage needed to operate the actuators. In principle this is not a fundamental consideration since voltages can readily be converted from the available battery potentials using transformers. These transformers need not sit within the propeller blades, where space is at a particular premium, but rather can be placed within the hull. However, the generation of the kilovolt level voltage that is required in all but the conducting polymer actuators requires relatively expensive components [13], which at present make the price objective impossible to achieve. As a result of voltage and cost considerations, conducting polymers appear to be the most promising actuator technology to meet the specifications.

Conducting Polymers: Challenges in Implementation. Work in fabricating and testing prototype variable camber foils [8] has demonstrated that a number of challenges need to be addressed if conducting polymer actuators are to be successfully applied to the actuation of variable camber propellers. Typical strains are on the order of $2 \%$, requiring large mechanical amplification. Electrolytes are employed, which may need to be encapsulated. In order to actuate with reasonable speeds the polymer must be made in thin films or fibres $(\sim 30 \mu \mathrm{m})$ to which good electrical contact is made and which are in contact with electrolyte. Based on results from initial prototype devices [8] these challenges can be overcome. 


\section{DISCUSSION AND CONCLUSION}

A major design challenge faced by potential users of electroactive polymers is determining which, if any, of the technologies is appropriate for a given application, what the advantages over established technologies are, and what the potential pitfalls may be in implementation. The large number of actuator technologies means that a general comparison is typically performed at a high level that can be difficult to translate for application to a real device [2]. In this paper a specific example application is chosen, and key figures of merit related to the application are determined, making comparison much simpler. The choice of actuator is narrowed down to four main candidates based primarily on work density, strain, and catch state. The final selection is made based on operating voltage and the costs associated with transforming potentials.

A number of qualitative comparisons are made in the process of deciding on appropriate actuators. For example, it is assumed that large strains are desirable as complexities of mechanical amplification increase with the magnitude of amplification needed. What magnitude of strain is too small to make amplification feasible? In making decisions on such issues it is really necessary to consider specific mechanisms, and these have not been considered here. Furthermore estimating the manufacturing and assembly costs of mechanical amplification will depend on the specific mechanism, and are thus difficult to generalize. In this regard the experience and judgement of the designer is relied upon, with actual implementation being the best indicator or success.

Work density has been chosen as a key figure of merit in this application, and this is one of the factors used to eliminate electric motors as strong contenders. Work density is based on work per stroke or actuator rotation and does not take into account the rate of actuation or the actuator power. It may be feasible, at the cost of some complexity, to operate fast actuators over many cycles in order to obtain the required work output within a small volume. For example if the work density of a rotary motor is $1 / 10^{\text {th }}$ of what is required to vary camber, it can be run through 10 rotations to produce the work, with the aid of a transmission system. Similarly a piezoceramic actuator and ratchet mechanism might be used to generate large displacements from the small strains. In selecting actuator figure of merit it is important not to inadvertently eliminate creative solutions using actuators that otherwise appear inappropriate. Are such alternatives feasible? Ultimately the designer needs to imagine appropriate mechanisms and perhaps even build prototypes in order to select the best approach.

Appropriate figures of merit will vary by application. The variable camber problem does not require fast response and thus strain rate and power density are not considered. However in situations where response must be obtained within several milliseconds or operational frequencies are in the tens of hertz or higher, power and rate become particularly important. In such cases actuators involving the motion of ions, such as conducting polymers, IPMCs and carbon nanotube actuators, are immediately at a disadvantage, with short ion travel distances and small internal resistances being key to obtaining rapid response. Actuators that are more directly field driven, such as ferroelectric polymers and dielectric elastomers, are in general simpler to operate at high frequencies. Although figures of merit vary by application, it is hoped that the example given and some of the specific challenges raised will help the designer in sorting through the large selection of actuators.

The variable camber foil chosen for this study has several features that make electroactive polymers (EAP) particularly attractive as actuators, and are general properties that can be used to flag promising EAP applications. The need to pack the actuator into a very confined space, necessitating high work density, is one factor that immediately suggests the use of EAPs. If low mass is important, then EAPs are even more favored because of their low densities. The need to hold position against a force also suggests EAPs, as many feature catch states. Currently only cycle lives of $<10$ million can be tolerated, as most actuators fail or have not been tested up to this point. Other indicators that suggest the use of EAPs are a need for low noise operation and, though this has yet to be fully explored, a requirement for biocompatibility.

\section{ACKNOWLEDGMENTS}

This work was performed at Molecular Mechanisms LLC as part of a U.S. Office of Naval Research sponsored STTR. 


\section{REFERENCES}

[1] Y. Bar-Cohen Ed., Smart Structure and Materials 2003, Electroactive Polymer Actuators and Devices, Proc. SPIE Vol. $5051,2003$.

[2] John D. Madden, Nate Vandesteeg, Patrick A. Anquetil, Peter G. Madden, Arash Takshi, Rachel R. Pytel, Serge R. Lafontaine, Paul A. Wieringa and Ian W. Hunter, "Artificial Muscle Technology: Physical Principles and Naval Prospects", IEEE Journal of Oceanic Engineering, in press.

[3] J. Hollerbach, I. Hunter \& J. Ballantyne "A comparative analysis of actuator technologies for robotics", O. Khatib, J. Craig \& Lozano-Perez Eds, The Robotics Review 2, MIT Press, Cambridge MA 1992, pp. 299-342.

[4] I. Hunter \& S. Lafontaine, "A comparison of muscle with artificial actuators", Technical Digest IEEE Solid State Sensors \& Actuators Workshop, 1992, pp. 178-185.

[5] K. MacLetchie et. al., IEEE Journal of Oceanic Engineering this issue.

[6] G. S. Triantafyllou, M. S. Triantafyllou and M. A. Grosenbaugh in Journal of Fluids and Structures, Vol. 7, No. 2, pages 205-224; 1993.

[7] Stanford et. al., "Electroactive polymer artificial muscle for underwater robotics", IEEE Journal of Oceanic Engineering, in press.

[8] John D.W. Madden, Bryan Schmid, Ralph Botha, Martin Hechinger, Serge R. Lafontaine, Peter G.A. Madden, Franz S. Hover, Karl McLetchie, Richard Kimball and Ian W. Hunter, "Application of Polypyrrole Actuators: Feasibility of Variable Camber Foils", IEEE Journal of Oceanic Engineering, in press.

[9] Online content: www.sippican.com, 2004.

[10] Online content available at http://www.futaba-rc.com/servos/futm0029.html. Note that electric motors can work if they are allowed to spin continuously at moderate to high speeds and have a mechanical catch to hold them in place when needed. The spinning approach requires gearing to convert the low torque, high revolution rate output into high torque and low revolution actions. An additional actuator is required to drive a latch mechanism.

[11] Hollerbach, J.M., Lang, J., Vaaler, E., Garabieta, I., Sepe, R., Umans, S., and Hunter, I.W., " The McGill/MIT Direct Drive Motor project," Proc. IEEE Intl. Conf. Robotics and Automation, May 2-7, 1993, Atlanta, pp. 2:611-617.

[12] S. Ashley, “Artificial Muscles", Scientific American, pp. 52-59, October 2003.

[13] EMCO High Voltage, Online content available at http://www.emcohighvoltage.com/ 2004. 